\title{
O tempo sentado está associado aos fatores de risco cardiometabólicos em adolescentes?
}

\section{Is sitting time associated with cardiometabolic risk factors in adolescents?}

\section{AUTORES \\ Géssika Castilho dos Santos ${ }^{1}$ (D) \\ Wagner de Campos ${ }^{2}$ (D) \\ Waynne Ferreira de Faria ${ }^{3}$ (D) \\ Jadson Marcio da Silva ${ }^{3}$ (D) \\ Rodrigo Bozza ${ }^{4}$ (D) \\ Luis Paulo Gomes Mascarenhas ${ }^{5}$ (iD \\ Anderson Zampier Ulbrich ${ }^{6}$ (D) \\ Antonio Stabelini $\mathrm{Neto}^{3}$ (D) \\ 1 Universidade Estadual de Londrina, Programa de Pós-Graduação Associado em Educação Física. Universidade Estadual de Maringá/Universidade Estadual de Londrina, Londrina, Paraná, Brasil. \\ 2 Universidade Federal do Paraná, Departamento de Educação Física, Curitiba, Paraná, Brasil. \\ 3 Universidade Estadual do Norte do Paraná, Centro de Ciências da Saúde, Jacarezinho, Paraná, Brasil. \\ 4 Universidade Positivo, Escola de Ciências da Saúde, Curitiba, Paraná, Brasil. \\ 5 Universidade Estadual do Centro-Oeste, Departamento de Educação Física, Irati, Paraná, Brasil. \\ 6 Universidade Federal do Paraná, Departamento de} Medicina Integrada, Curitiba, Paraná, Brasil.

\section{CONTATO}

Géssika Castilho dos Santos gessika.castilho@gmail.com

Universidade Estadual do Norte do Paraná, Centro de Ciências da Saúde,

Alameda Padre Magno, no 841

Nova Jacarezinho, Jacarezinho, Paraná, Brasil. CEP: 86400-000.

$\mathrm{DOI}$

$10.12820 /$ rbafs. $25 \mathrm{e} 0132$

\begin{abstract}
RESUMO
O presente estudo teve como objetivo verificar a associação entre o tempo sentado e fatores de risco cardiometabólicos em adolescentes. A amostra foi composta por 454 adolescentes (50,6\% masculino) com idades entre 12 a 18 anos. O tempo diário despendido sentado e a prática de atividade física moderada-vigorosa foram avaliados por meio do recordatório de três dias de Bouchard. Os fatores de risco cardiometabólicos analisados foram: circunferência de cintura; glicose; HDL-C; triglicerídeos; e pressão arterial. Foi utilizada a análise de variância com dois fatores (sexo e quartis) para comparar os fatores de risco cardiometabólicos entre os quartis de tempo sentado. Para verificar a associação entre o tempo sentado e os fatores de risco cardiometabólicos foi empregado a regressão linear múltipla. Foi observado que os meninos pertencentes ao $4^{\circ}$ quartil (maior tempo sentado) apresentaram valores mais elevados de glicose e triglicerídeos em comparação com seus pares com menos tempo. No sexo feminino, verificou-se que as pertencentes ao $3^{\circ}$ e $4^{\circ}$ quartil apresentaram maiores valores de triglicerídeos e glicose em comparação ao $1^{\circ}$ quartil. Associações positivas e significativas foram observadas entre o tempo sentado e os valores de circunferência da cintura $(\beta=0,093 ; p<0,05)$, glicose $(\beta=0,111 ; \mathrm{p}<0,05)$ e pressão arterial diastólica $(\beta=-0,115 ; \mathrm{p}<0,05)$ mesmo após o ajuste para a prática de atividade física moderada-vigorosa. De acordo com os achados do presente estudo, conclui-se que os adolescentes que permaneceram maior tempo sentado apresentaram maiores valores de glicose, triglicerídeos e escore de risco cardiometabólico em comparação aos que despenderam menos tempo.
\end{abstract}

Palavras-chave: Comportamento sedentário; Adolescente; Perfil metabólico; Estilo de vida.

\section{ABSTRACT}

This study aimed to verify if there is an association between sitting time and cardiometabolic risk factors in adolescents. The sample was composed of 454 adolescents (50.6\% male) aged between 12 and 18 years. The time spent in sitting and physical activity were assessed through a record of three days by Bouchard. The following cardiometabolic risk factors were analyzed: waist circumference, glucose, $H D L$ - cholesterol, triglycerides, and blood pressure. It was used the Two-way Analysis of Variance (sex and quartiles) to compare the cardiometabolic risk factors between the quartiles of sitting time. To verify the association between sitting time and cardiometabolic risk factors it was used the multivariate linear regression. Boys of the 4th quartile (higher sitting time) presented higher values of glucose and triglycerides compared to their peers with less time. Girls of the 3rd and 4th quartile presented higher triglycerides and glucose values, compared to the 1st quartile. Significant associations $(p<0.05)$ were observed between sitting time with waist circumference $(\beta=0.093 ; p<0.05)$, glucose $(\beta=0.111 ; p<0.05)$ and diastolic blood pressure $(\beta=0.115 ; p<0.05)$ even after the adjustment for the moderate to vigorous physical activity. According to the findings of this study, we concluded that adolescents who spent more sitting time had higher values of glucose, triglycerides and cardiometabolic risk score compared with who has less sitting time.

Keywords: Sedentary behavior; Adolescent; Metabolic profile; Lifestyle.

\section{(cc) BY}

Este trabalho está licenciado com uma Licença

Creative Commons - Atribuição 4.0 Internacional.

\section{Introdução}

Os avanços tecnológicos têm permitido aos usuários acesso a inúmeras experiências e informações de forma rápida por meio de dispositivo móvel. Este processo substitui a necessidade de movimento, tornando a sociedade moderna cada vez mais sedentária ${ }^{1}$. Entre adolescentes, as opções tecnológicas são um atrativo que resulta em um gasto no tempo de tela acima das recomendações ${ }^{2}$.

O comportamento sedentário é caracterizado por 
um conjunto de atividades realizadas na posição senta$\mathrm{da}$, deitada ou inclinada que requerem um gasto energético abaixo de 1,5 $\mathrm{METs}^{3}$. Em adultos, existe forte evidência de que elevado tempo diário despendido em comportamento sedentário aumenta o risco de mortalidade por todas as causas e mortalidade por doenças cardiovasculares (DCV), bem como aumenta a incidência de DCV e diabetes tipo $2^{4}$.

Pesquisas sugerem que o comportamento sedentário adotado por crianças e adolescentes pode estar associado a fatores de risco para doenças cardiometabólicas ${ }^{5}$ Em revisão realizada por Rezende et al. ${ }^{6}$, os autores verificaram em crianças e adolescentes uma forte evidência da relação entre o tempo gasto em comportamento sedentário e obesidade e uma evidência moderada foi observada para pressão arterial, colesterol total, autoestima, problemas de comportamento social, desempenho acadêmico e aptidão física. Em adição, Tremblay et al. ${ }^{7}$ demonstraram que o aumento do tempo em atividades sedentárias esteve positivamente associado ao aumento de massa gorda, do índice de massa corporal, da insulina em jejum e da resistência à insulina. Nightingale et $\mathrm{al}^{8}$ verificaram uma associação positiva entre o tempo de tela maior ou igual a 3 horas diárias com a obesidade infantil.

$\mathrm{Na}$ literatura, há divergência entre estudos quando se refere ao efeito isolado do comportamento sedentário sobre a saúde de adolescentes. Alguns autores ${ }^{9,10}$ indicaram uma associação positiva entre o tempo gasto em comportamento sedentário e os fatores de risco cardiometabólicos (índice de massa corporal, circunferência de cintura, glicemia, lipoproteína de baixa densidade, triglicerídeos e insulina) em adolescentes independente da prática de atividade física moderada a vigorosa (AFMV), enquanto outros ${ }^{11,12}$ sugerem que altos níveis de prática de atividade física parecem atenuar as consequências negativas do comportamento sedentário, sugerindo que mais pesquisas são necessárias para melhorar o entendimento sobre as interações entre atividade física/comportamento sedentário nos desfechos de saúde.

Desta forma, ainda permanecem inconclusivas as inter-relações entre o tempo gasto em comportamento sedentário e os fatores de risco para doenças cardiometabólicas em adolescentes, principalmente quando se considera a prática de AFMV. Portanto, o objetivo deste estudo foi verificar a associação entre o tempo sentado e os fatores de risco para doenças cardiometabólicas isolados e agrupados em adolescentes.

\section{Métodos}

Trata-se de um estudo com delineamento transversal conduzido com adolescentes da cidade de Curitiba, Paraná. A seleção da amostra foi feita a partir de um processo de amostragem probabilística por conglomerados em dois estágios (Estágio 1 - escolas sorteadas por região geográfica; Estágio 2 - Turmas sorteadas por escola), composta por adolescentes escolares de ambos os sexos, com idades entre 10-18 anos, matriculados na rede de ensino pública da cidade. A população total correspondia a 223.285 escolares segundo informações da Secretaria Municipal de Educação. O tamanho amostral foi calculado de acordo com os seguintes critérios: 1) número total de meninos e meninas entre 10-18 anos, da cidade de Curitiba; 2) intervalo de confiança de 95\%; e 3) erro amostral de cinco pontos percentuais e prevalência de $50 \%$. A escolha da prevalência de $50 \%$ foi adotada, pois o desfecho HDL-C baixo é o fator de risco com prevalência mais elevada em estudos com adolescentes (aproximadamente 50\%) ${ }^{13}$. A amostra foi estimada em 383 adolescentes. A esta estimativa foi adicionado o efeito do desenho de 1,2 como correção ao delineamento amostral por conglomerados ${ }^{14}$, totalizando 460 adolescentes como amostra mínima. Dos 472 adolescentes que estavam nas salas sorteadas no dia da avaliação, 18 recusaram a participar por motivos pessoais ou foram excluídos, pois não atenderem os critérios estabelecidos previamente: 1) tabagismo; 2) apresentar história familiar de doença cardíaca; 3) diabético; e 4) estar ingerindo medicamento no dia do teste. A amostra final foi constituída por 454 adolescentes, sendo 230 (50,6\%) do sexo masculino e 224 do sexo feminino. A pesquisa foi aprovada pelo Comitê de Ética em Pesquisa da Universidade Federal do Paraná (protocolo: 018-06), de acordo com a resolução 196/96 do Conselho Nacional de Saúde.

Todos os procedimentos de coletas de dados, com exceção da coleta sanguínea, foram realizados por pesquisadores devidamente treinados e foram utilizadas técnicas padronizadas para a coleta de todas as variáveis do estudo. Os dados foram coletados nas próprias escolas participantes do estudo, no período da manhã, de agosto a novembro de 2008. Foram realizadas avaliações duplicadas em todas as variáveis, considerando-se o valor médio entre as duas mensurações. Caso as medidas diferissem mais que $3 \mathrm{~cm}$ para estatura, $0,1 \mathrm{~kg}$ para a massa corporal, $1 \mathrm{~cm}$ para circunferência de cintura e $2 \mathrm{mmHg}$ para pressão arterial, uma terceira avaliação era realizada. 
A estatura foi avaliada por meio de um estadiômetro vertical portátil escalonado em $0,1 \mathrm{~cm}$. O cursor do aparelho foi colocado no ponto mais alto da cabeça com o avaliado em apnéia inspiratória. Para determinar a massa corporal foi utilizada uma balança digital portátil com resolução de $100 \mathrm{~g}$. O índice de massa corporal (IMC) foi calculado por meio da fórmula: [massa corporal $(\mathrm{kg}) /$ estatura $\left.(\mathrm{m})^{2}\right]$.

A circunferência da cintura foi mensurada no ponto médio entre o último arco costal e a crista ilíaca, sendo que sujeito deveria estar confortavelmente na posição ereta, com as mãos ao lado do corpo, com o abdômen relaxado e vestindo o mínimo de roupas que pudesse obstruir a identificação do local e realização da medida.

Foram coletados aproximadamente $8 \mathrm{ml}$ de sangue de cada adolescente com um jejum prévio de 12 horas para análises bioquímicas. A coleta do sangue foi realizada por enfermeiras do próprio laboratório onde as amostras sanguíneas foram analisadas, no período das 7:00 às 9:00 horas da manhã. Para análise laboratorial e determinação da glicose $(\mathrm{mg} / \mathrm{dL})$, da lipoproteína de alta densidade - colesterol (HDL-C) e triglicerídeos (TG) foi usado o método enzimático-colorimétrico automatizado através do aparelho da marca ABBOTT SPECTRUM, modelo CCX.

A pressão arterial foi mensurada através do método auscultatório, seguindo os parâmetros estabelecidos pelo National High Blood Pressure Education Program ${ }^{15}$. A pressão arterial sistólica (PAS) e diastólica (PAD) foram mensuradas no braço direito do avaliado, utilizando um esfigmomanômetro aneróide e estetoscópio (Rappaport Premium), após o adolescente permanecer sentado em repouso por um período de 5 minutos. A pressão arterial sistólica foi definida como o som de Korotkoff fase 1 e a diastólica como o som de Korotkoff fase 5.

Para cada fator de risco foi calculado o escore $\mathrm{Z}$ (valor individual - média da amostra / desvio padrão da amostra). Para pressão arterial foi utilizada a média da PAS e PAD (PAS + PAD / 2) para cálculo do escore $Z$. Para o HDL-C, como, ao contrário dos demais componentes, um valor baixo significa um resultado desfavorável, o cálculo do escore foi invertido (média da amostra - valor individual/ desvio padrão da amostra). A somatória do valor de escore $\mathrm{Z}$ de cada componente representou o Escore de risco cardiometabólico (Escore $Z$ total $=$ Escore $Z$ cintura + Escore $Z$ pressão arterial + Escore Z glicemia + Escore Z HDL-C + Escore $\mathrm{Z}$ triglicerídeos). Esta metodologia para criação de um índice de risco metabólico agrupado tem sido descrita previamente em outros estudos com adolescentes ${ }^{16,17}$.

O estágio de maturação sexual foi avaliado por meio do método proposto por Tanner ${ }^{18}$, no qual os estágios maturacionais se dividem de 1 a 5 . O exame foi aplicado na forma de auto avaliação da pilosidade pubiana.

A atividade física e o tempo sentado foram avaliadas pelo recordatório de três dias de Bouchard ${ }^{19}$ ( 2 dias na semana: terça-feira e quinta-feira e 1 dia de final de semana: domingo), composto por nove categorias: 1 (sono), 2 (atividades sentado); 3-5 (atividade leve); 6-8 (moderada) e 9 (vigorosa), as quais são divididas em períodos de 15 minutos. Neste recordatório de auto relato, o adolescente preencheu referente às atividades realizadas durante os períodos de tempo específicos. $\mathrm{O}$ instrumento foi preenchido em sala de aula com orientação do pesquisador. Os adolescentes foram orientados a registrar o tipo de atividade realizada em cada período de 15 minutos ao longo das 24 horas do dia. $\mathrm{O}$ registro das informações foi referente a semana anterior a aplicação do teste.

O tempo gasto sentado foi determinado pela categoria 2, expressa em minutos por dia, e os adolescentes foram classificados em quartis (10 Quartil: menor tempo sedentário e $4^{\circ}$ quartil: maior tempo sedentário). $\mathrm{O}$ tempo gasto em AFMV foi determinado pela somatória das categorias 6-9. Este questionário apresenta reprodutibilidade de $\mathrm{r}=0,91$ em indivíduos a partir de 10 anos de idade ${ }^{19}$ e foi validado para utilização em adolescentes através da técnica de água duplamente marcada $(0,54)^{20}$ e pelo acelerômetro $(0,33-0,35)^{21}$.

A normalidade de distribuição dos dados foi verificada pelo teste de Kolmogorov Smirnov, em seguida o teste $t$ de Student para grupos independentes. Para os dados que não apresentaram distribuição normal, foram empregadas transformações logarítmicas. Para comparações dos fatores de risco cardiometabólicos entre os quartis de tempo sentado foi utilizada a análise de variância (ANOVA) com dois fatores (sexo e quartis), após a verificação do teste de igualdade dos erros das variâncias (Levene). Quando este pressuposto não foi atendido, transformações logarítmicas foram aplicadas (CC, glicose, HDL e triglicerídeos). Caso o teste $\mathrm{F}$ fosse estatisticamente significante, as comparações múltiplas foram efetuadas pelo teste de Bonferroni. Para verificar a associação entre o tempo sentado e as medidas de interesse foi empregada a regressão linear múltipla. Modelo 1 - bruto; Modelo 2 - ajustado por sexo, idade e maturação sexual; Modelo 3 - ajuste 
adicional para o IMC; e Modelo 4 - ajuste adicional para a prática de atividade física moderada-vigorosa. A análise do resíduo foi realizada e, para cada modelo, a suposição de homocedasticidade e distribuição normal foi seguida. A avaliação de multicolineariedade entre as variáveis independentes foram testadas a partir dos valores de tolerância $(>0,1)$ e VIF $(<10)$. Para análise dos dados foi utilizado o pacote estatístico SPSS versão 22.0, a significância foi estipulada em $\mathrm{p}<0,05$.

\section{Resultados}

As características descritivas dos adolescentes podem ser observadas na Tabela 1 . Os meninos apresentaram maior circunferência de cintura, glicemia, pressão arterial sistólica, maior tempo sentado $(491,41 \pm 168,20$ $\mathrm{min} /$ dia vs. 461,52 $\pm 161,19 \mathrm{~min} /$ dia; $\mathrm{p}<0,05)$ e prática de atividade física moderada a vigorosa $(113,48$ $\pm 85,02 \mathrm{~min} /$ dia vs. $67,88 \pm 79,19 \mathrm{~min} / \mathrm{dia} ; \mathrm{p}<0,05)$ quando comparado as meninas. Já as meninas apresentaram maiores índices de lipoproteína de alta densidade (HDL-C).

Tabela 1 - Comparação da idade, tempo sentado, atividade física e fatores de risco cardiometabólico entre os sexos (Curitiba; $\mathrm{n}=454$ ).

\begin{tabular}{lccc}
\hline Variável & $\begin{array}{c}\text { Meninos } \\
(\mathrm{n}=230)\end{array}$ & $\begin{array}{c}\text { Meninas } \\
(\mathrm{n}=224)\end{array}$ & $\begin{array}{c}\text { Total } \\
(\mathrm{n}=454)\end{array}$ \\
\hline Idade $(\mathrm{anos})$ & $14,70 \pm 1,69$ & $14,58 \pm 1,67$ & $14,64 \pm 1,68$ \\
$\mathrm{IMC}\left(\mathrm{Kg} / \mathrm{m}^{2}\right)$ & $20,31 \pm 2,95$ & $20,46 \pm 3,73$ & $20,39 \pm 3,35$ \\
CC $(\mathrm{cm})$ & $70,94 \pm 8,44^{*}$ & $65,62 \pm 7,03$ & $68,32 \pm 8,21$ \\
Glicose (mg/dl) & $89,47 \pm 13,71^{*}$ & $84,33 \pm 14,02$ & $86,93 \pm 14,09$ \\
HDL-C (mg/dl) & $45,60 \pm 11,00^{*}$ & $50,06 \pm 14,10$ & $47,80 \pm 12,81$ \\
Triglicerídeos (mg/dl) & $88,48 \pm 39,09$ & $85,87 \pm 33,88$ & $87,19 \pm 36,60$ \\
PAS (mmHg) & $101,00 \pm 14,13^{*}$ & $95,48 \pm 13,56$ & $98,28 \pm 14,11$ \\
PAD (mmHg) & $69,16 \pm 10,51$ & $68,25 \pm 10,97$ & $68,71 \pm 10,74$ \\
Z escore geral & $0,23 \pm 2,94$ & $0,27 \pm 2,68$ & $0,25 \pm 2,81$ \\
Tempo Sentado & $491,41 \pm 168,20^{*}$ & $461,52 \pm 161,19$ & $476,66 \pm 165,28$ \\
(min/dia) & & & \\
AFMV (min/dia) & $113,48 \pm 85,02^{*}$ & $67,88 \pm 79,19$ & $90,98 \pm 85,22$ \\
\hline
\end{tabular}

$\mathrm{IMC}=$ índice de massa corporal; $\mathrm{CC}=$ circunferência de cintura; HDL-C = lipoproteína de alta densidade; $\mathrm{PAS}$ = pressão arterial sistólica; $\mathrm{PAD}=$ pressão arterial diastólica; $\mathrm{AFMV}=$ atividade física de moderada a vigorosa. ${ }^{*}$ p < 0,05 em comparação às meninas; Dados estão descritos em média \pm desvio-padrão.

$\mathrm{Na}$ Tabela 2 são apresentadas as informações referentes aos fatores de risco cardiometabólicos de acordo com os quartis de tempo sentado. Nota-se que os meninos pertencentes ao $4^{\circ}$ quartil apresentaram níveis mais elevados de glicose $(91,68 \pm 12,73 \mathrm{mg} / \mathrm{dl}$ vs. 86,10 $\pm 13,48 \mathrm{mg} / \mathrm{dl})$ e triglicerídeos $(102,48 \pm 2,49 \mathrm{mg} / \mathrm{dl}$ vs.
$82,44 \pm 31,89 \mathrm{mg} / \mathrm{dl}$ ) em comparação com seus pares do $1^{\circ}$ quartil. No sexo feminino, as pertencentes ao $3^{\circ} \mathrm{e}$ $4^{\circ}$ quartil de tempo sentado apresentaram valores mais elevados de triglicerídeos e glicose, respectivamente. Em relação ao escore de risco cardiometabólico, em ambos os sexos, adolescentes que estavam no $4^{\circ}$ quartil apresentaram um maior escore de risco cardiometabólico em comparação ao $2^{\circ}$ quartil.

Associações positivas e significativas foram observadas entre o tempo gasto sentado e os valores de circunferência da cintura, glicose e pressão arterial diastólica (Tabela 3). Estas associações permaneceram significativas após ajuste para idade, sexo e maturação sexual (modelo 2). Quando foi adicionado o IMC (modelo 3), apenas a associação com a circunferência da cintura perdeu a significância. Após o ajuste pela prática de atividade moderada-vigorosa (modelo 4), as associações iniciais (análise bruta) permaneceram estatisticamente significantes.

\section{Discussão}

A presente pesquisa verificou associação significativa entre o tempo sentado e os fatores de risco para doenças cardiometabólicas em adolescentes mesmo após o ajuste pela prática de atividade física moderada-vigorosa. Os resultados sugerem que o tempo gasto em comportamento sedentário foi associado aos fatores de risco isolados ( $\mathrm{CC}$, glicose e $\mathrm{PAD})$, porém não com o escore de risco cardiometabólico. Em relação a análise de comparação entre os sexos, foi observado que os meninos gastaram mais tempo diário em atividades sedentárias e em atividades físicas de intensidade moderada-vigorosa quando comparado ao sexo feminino, condizente com os resultados de pesquisas prévias ${ }^{22,23}$.

Quando analisado a comparação do escore de risco cardiometabólico entre os quartis de tempo sentado, observou-se que em ambos os sexos, os adolescentes que gastaram mais tempo em atividades sedentárias apresentaram um maior escore de risco cardiometabólico agrupado em comparação com seus pares que passaram menos tempo nestas atividades, sugerindo que os padrões hipocinéticos repercutem de maneira negativa na saúde deste grupo populacional.

Ao analisar de forma isolada os fatores de risco entre os quartis do tempo sentado, foi observado que independente do sexo, os adolescentes com maior comportamento sedentário apresentaram níveis mais elevados de triglicerídeos e glicose, e maiores valores de circunferência da cintura apenas para os meninos. Canabrava 
Tabela 2 - Comparação dos fatores de risco cardiometabólico entre os quartis de tempo sentado.

\begin{tabular}{|c|c|c|c|c|}
\hline & $1^{\circ}$ Quartil & $2^{\circ}$ Quartil & $3^{\circ}$ Quartil & $4^{\circ}$ Quartil \\
\hline \multicolumn{5}{|c|}{ Meninos (n=230) } \\
\hline $\mathrm{CC}(\mathrm{cm})$ & $69,52 \pm 7,00$ & $69,71 \pm 6,98$ & $72,48 \pm 9,11^{*}$ & $71,53 \pm 9,69$ \\
\hline Glicose (mg/dl) & $86,10 \pm 13,48$ & $88,60 \pm 14,03$ & $90,57 \pm 14,28$ & $91,68 \pm 12,73^{\#}$ \\
\hline HDL-C (mg/dl) & $46,48 \pm 11,71$ & $45,30 \pm 10,16$ & $46,23 \pm 11,37$ & $44,54 \pm 10,90$ \\
\hline Triglicerídeos (mg/dl) & $82,44 \pm 31,89$ & $88,02 \pm 33,58$ & $79,97 \pm 29,20$ & $102,48 \pm 2,49^{* *}$ \\
\hline PAS (mmHg) & $98,31 \pm 12,96$ & $100,89 \pm 15,26$ & $102,52 \pm 14,18$ & $101,60 \pm 13,94$ \\
\hline $\mathrm{PAD}(\mathrm{mmHg})$ & $68,58 \pm 11,60$ & $67,80 \pm 10,61$ & $70,43 \pm 10,78$ & $69,47 \pm 9,25$ \\
\hline Escore de risco metabólico & $-0,47 \pm 2,66$ & $-0,09 \pm 2,77$ & $0,29 \pm 3,11$ & $1,00 \pm 2,99^{* *}$ \\
\hline \multicolumn{5}{|c|}{ Meninas $(\mathrm{n}=224)$} \\
\hline $\mathrm{CC}(\mathrm{cm})$ & $65,30 \pm 6,81$ & $65,22 \pm 6,27$ & $64,92 \pm 7,21$ & $67,20 \pm 7,91$ \\
\hline Glicose (mg/dl) & $81,95 \pm 10,44$ & $82,53 \pm 13,52$ & $90,57 \pm 14,28$ & $91,68 \pm 12,73^{*} \#$ \\
\hline HDL-C (mg/dl) & $49,07 \pm 15,19$ & $51,08 \pm 14,86$ & $49,95 \pm 14,18$ & $50,30 \pm 11,75$ \\
\hline Triglicerídeos (mg/dl) & $84,90 \pm 33,40$ & $79,13 \pm 29,29$ & $93,32 \pm 36,17^{\#}$ & $87,82 \pm 36,50$ \\
\hline PAS (mmHg) & $98,30 \pm 13,98^{\# ¥}$ & $91,31 \pm 13,54$ & $93,18 \pm 12,69$ & $98,92 \pm 12,38^{\# ¥}$ \\
\hline $\mathrm{PAD}(\mathrm{mmHg})$ & $72,97 \pm 12,02^{\# \nVdash e}$ & $64,98 \pm 10,42$ & $65,63 \pm 10,49$ & $68,44 \pm 8,39$ \\
\hline Escore de risco metabólico & $0,47 \pm 2,68$ & $-0,47 \pm 2,79$ & $0,29 \pm 2,43$ & $0,88 \pm 2,66^{\#}$ \\
\hline
\end{tabular}

$\mathrm{CC}=$ circunferência de cintura; HDL = lipoproteína de alta densidade; $\mathrm{PAS}=$ pressão arterial sistólica; $\mathrm{PAD}=$ pressão arterial diastólica. ${ }^{*} \mathrm{p}<$ 0,05 em relação ao $1^{\circ}$ quartil, \#p $<0,05$ em relação ao $2^{\circ}$ quartil, ¥p $<0,05$ em relação ao $3^{\circ}$ quartil, €p $<0,05$ em relação ao $4^{\circ}$ quartil.

Tabela 3 - Associação entre o tempo sentado e os fatores de risco cardiometabólico individuais e agrupado.

\begin{tabular}{lcccc}
\hline & Modelo 1 & Modelo 2 & Modelo 3 & Modelo 4 \\
\hline Cintura & $0,127(0,010 ; 0,060)^{*}$ & $0,113(0,007 ; 0,054)^{*}$ & $0,026(-0,006 ; 0,020)$ & $0,093(0,000 ; 0,050)^{*}$ \\
Glicose & $0,171(0,030 ; 0,100)^{*}$ & $0,154(0,025 ; 0,093)^{*}$ & $0,139(0,019 ; 0,087)^{*}$ & $0,111(0,005 ; 0,081)^{*}$ \\
HDL-C & $0,026(-0,040 ; 0,072)$ & $0,035(-0,034 ; 0,077)$ & $0,048(-0,027 ; 0,085)$ & $-0,017(-0,070 ; 0,050)$ \\
Triglicerídeos & $0,069(-0,020 ; 0,142)$ & $0,064(-0,025 ; 0,138)$ & $0,045(-0,042 ; 0,121)$ & $0,047(-0,048 ; 0,133)$ \\
PAS & $0,016(-0,025 ; 0,036)$ & $0,005(-0,029 ; 0,032)$ & $-0,030(-0,039 ; 0,019)$ & $-0,003(-0,035 ; 0,033)$ \\
PAD & $-0,134(-0,083 ;-0,015)^{*}$ & $-0,131(-0,082 ;-0,015)^{*}$ & $-0,169(-0,094 ;-0,030)^{*}$ & $-0,115(-0,079 ;-0,006)^{*}$ \\
Escore de risco metabólico & $0,075(-0,269 ; 2,505)$ & $0,085(-0,107 ; 2,663)$ & $0,022(-0,826 ; 1,495)$ & $0,070(-0,466 ; 2,564)$ \\
\hline
\end{tabular}

Modelo 1. Análise sem ajustes (bruto); Modelo 2. Ajuste pelo sexo, idade e maturação; Modelo 3. Ajuste adicional pelo IMC; Modelo 4. Ajuste adicional pela AFMV. Dados estão apresentado em beta coeficientes padronizados (intervalo de confiança de $95 \%$ ). HDL-C = lipoproteína de alta densidade; $\mathrm{PAS}=$ pressão arterial sistólica; $\mathrm{PAD}=$ pressão arterial diastólica. ${ }^{*} \mathrm{p}<0,05$.

et al. ${ }^{24}$ demonstraram em sua revisão sistemática que o comportamento sedentário está associado positivamente a fatores de risco para doenças cardiovasculares em crianças e adolescentes (glicemia, insulina, resistência à insulina, LDL-C e triglicerídeos).

Estes achados sugerem que permanecer longos períodos de tempo em situações com gasto energético < 1,5 METS gera impacto negativo no perfil cardiometabólico. Essas consequências podem ser explicadas por meio das alterações fisiológicas e metabólicas causadas por períodos prolongados em atividades hipocinéticas. Young et al. ${ }^{25}$ explicam que o tempo gasto em atividades sedentárias diminui a utilização de substratos energéticos derivados da gordura, particularmente a oxidação de ácidos graxos provenientes da hidrólise de triglicerídeos tanto no musculoesquelético como no tecido adiposo, causando o aumento dos triglicerídeos no organismo. Além disso, esse comportamento ocasiona uma diminuição na atividade celular, levando a um aumento da resistência à insulina, diminuição da expressão e da atividade dos transportadores de glicose e uma menor utilização da glicose como substrato energético, resultando assim em um aumento dos índices glicêmicos ${ }^{26}$.

Discussões acadêmicas recentes vêm questionando se os efeitos deletérios à saúde pelo comportamento sedentário são independentes da prática de atividade física. No presente estudo, o tempo gasto em comportamento sedentário foi associado positivamente com a circunfe- 
rência de cintura, glicose e inversamente com a pressão arterial diastólica, sendo essa associação mantida quando o modelo da regressão foi ajustado pela prática de atividade física moderada-vigorosa. Estes achados são consistentes com evidências prévias que mostram que o comportamento sedentário e a atividade física devem ser considerados comportamentos independentes quando o objetivo é investigar o risco à saúde 9,27,28-30. $^{\text {. }}$

Fröberg \& Raustorp ${ }^{31}$ realizaram uma revisão sistemática e demonstraram que as associações entre o tempo gasto em atividades sedentárias e risco cardiometabólico individual e agrupado em jovens é fraca quando o tempo gasto em AFMV é considerado nas análises. No presente estudo, houve uma redução da magnitude da associação entre o tempo sentado e os fatores de risco cardiometabólicos após incluir a AFMV na análise, contudo essas associações permaneceram significativas.

Evidências científicas ${ }^{16,32}$ vêm enfatizando os benefícios obtidos pela prática regular de AF, principalmente em relação a prevenção no desenvolvimento de doenças crônico-degenerativas em crianças e adolescentes. Todavia, segundo o Comitê de Diretrizes de Atividade Física dos Estados Unidos ${ }^{33}$, os efeitos benéficos da AFMV sobre a mortalidade por todas as causas variam de acordo com a quantidade de tempo gasto em comportamento sedentário ${ }^{33}$.

Nossos resultados apresentam algumas implicações práticas que reforçam a importância de elaborar e adotar estratégias, tais como: incentivar os jovens a substituir parte do tempo sedentário com atividades físicas leves e posteriormente avançar para atividades de intensidade moderada; proporcionar locais e projetos que estimulem os adolescentes a se engajar em atividades físicas de intensidade moderada à vigorosa no ambiente escolar e no tempo livre; e desenvolver programas que estimulem a realização de interrupções no tempo sedentário (breaks), evitando permanecer por longos períodos sentados de forma contínua.

Algumas limitações do presente estudo devem ser destacadas, como o design transversal do estudo que não permite estabelecer uma relação de causa e efeito. Os métodos auto-reportados apresentam validade comprovada e são amplamente utilizados para avaliar a prática de atividade física e o comportamento sedentário em adolescentes, porém estes métodos estão suscetíveis ao viés de memória do avaliado. Além disso, ainda que o recordatório de três dias de Bouchard ${ }^{19}$ apresente uma categoria que avalia o tempo diário gasto sentado, o mesmo foi validado para avaliar a prática de ativi- dade física e não comportamento sedentário, devendo ser pontuado como uma limitação do presente estudo. Os pontos fortes do estudo devem ser ressaltados, tais como: as análises de regressão múltipla ajustadas por potenciais fatores de confusão incluindo atividade física moderada-vigorosa, maturação sexual e índice de massa corporal. Outro ponto forte é ter utilizado uma medida do tempo sentado e não apenas tempo de tela, uma vez que ainda são poucos os estudos que utilizaram esta abordagem para avaliação do comportamento sedentário em uma amostra representativa de adolescentes.

Diante disso, estudos futuros são necessários para analisar a relação de formas específicas do comportamento sedentário (tempo sentado não recreativo, tempo sentado recreativo e tempo de tela) com os desfechos à saúde na população infanto-juvenil.

Em conclusão, o tempo sentado foi associado com os fatores de risco cardiometabólicos em adolescentes mesmo ajustado pela prática de AFMV. Ademais, os adolescentes que despenderam mais tempo sentado apresentaram maiores valores de glicose, triglicerídeos e escore de risco cardiometabólico em comparação aos que despenderam menos tempo.

\section{Conflito de interesse}

Os autores declaram não haver conflito de interesses.

\section{Contribuição dos autores}

Santos GC, participou da elaboração do manuscrito. Campos W, participou da supervisão da coleta de dados. Faria WF. realizou a análise estatística. Silva JM, participou da elaboração do manuscrito. Bozza R, participou da coleta de dados. Ulbrich AZ, participou da coleta de dados. Mascarenhas LPG, participou da coleta de dados. Stabelini Neto A, participou da elaboração e correção final do manuscrito.

\section{Agradecimentos}

Os autores gostariam de agradecer ao Centro de Estudos em Atividade Física e Saúde (CEAFS) pelo seu apoio durante a coleta de dados referente a esse estudo.

\section{Referências}

1. Sousa GR, Silva DAS. Sedentary behavior based on screen time: prevalence and associated sociodemographic factors in adolescents. Cien Saude Colet. 2017;22(12):4061-72.

2. Henderson M, Benedetti A, Barnett TA, Mathieu ME, Deladoey J, Gray-Donald K. Influence of Adiposity, Physical Activity, Fitness, and Screen Time on Insulin Dynamics Over 2 Years in Children. JAMA Pediatr. 2016;170(3):227-35.

3. Tremblay MS, Aubert S, Barnes JD, Saunders TJ, Carson V, Latimer-Cheung AE, et al. Sedentary Behavior Research Network (SBRN) - Terminology Consensus Project process and outcome. Int J Behav Nutr Phys Act. 2017;14(1):1-17. 
4. Katzmarzyk PT, Powell KE, Jakicic JM, Troiano RP, Piercy K, Tennant B. Sedentary Behavior and Health: Update from the 2018 Physical Activity Guidelines Advisory Committee. Med Sci Sports Exerc. 2019;51(6):1227-41.

5. Cliff DP, Okely AD, Burrows TL, Jones RA, Morgan PJ, Collins CE, et al. Objectively measured sedentary behavior, physical activity, and plasma lipids in overweight and obese children. Obesity (Silver Spring). 2013;21(2):382-5.

6. Rezende LFM, Lopes MR, Rey-Lopez JP, Matsudo VKR, Luiz OC. Sedentary behavior and health outcomes: an overview of systematic reviews. PLoS One. 2014;9(8):e105620.

7. Tremblay MS, LeBlanc AG, Kho ME, Saunders TJ, Larouche $\mathrm{R}$, Colley RC, et al. Systematic review of sedentary behaviour and health indicators in school-aged children and youth. Int J Behav Nutr Phys Act. 2011;8:98.

8. Nightingale CM, Rudnicka AR, Donin AS, Sattar N, Cook DG, Whincup PH, et al. Screen time is associated with adiposity and insulin resistance in children. Arch Dis Child. 2017;102(7):612-6.

9. Leiva AM, Martinez MA, Cristi-Montero C, Salas C, Ramirez-Campillo R, Diaz Martinez X, et al. Sedentary lifestyle is associated with metabolic and cardiovascular risk factors independent of physical activity]. Rev Med Chil. 2017;145(4):458-67.

10. Saunders TJ, Chaput JP, Tremblay MS. Sedentary behaviour as an emerging risk factor for cardiometabolic diseases in children and youth. Can J Diabetes. 2014;38(1):53-61.

11. Skrede T, Steene-Johannessen J, Anderssen SA, Resaland GK, Ekelund U. The prospective association between objectively measured sedentary time, moderate-to-vigorous physical activity and cardiometabolic risk factors in youth: a systematic review and meta-analysis. Obes Rev. 2019;20(1):55-74.

12. Lavie CJ, Ozemek C, Carbone S, Katzmarzyk PT, Blair SN. Sedentary Behavior, Exercise, and Cardiovascular Health. Circ Res. 2019;124(5):799-815.

13. Stabelini Neto A, Bozza R, Ulbrich AZ, Vasconcelos ÍQA, Mascarenhas LPG, Boguszewski MCS, et al. Fatores de risco para aterosclerose associados à aptidão cardiorrespiratória e ao IMC em adolescentes. Arq Bras Endocrinol Metabol. 2008;52(6):1024-30.

14. Luiz RR, Magnanini MMF. A lógica da determinação do tamanho da amostra em investigações epidemiológicas. Cad. Saude Colet. 2000;8(2):9-28.

15. NHBPEP. Update on the 1987 Task Force Report on High Blood Pressure in Children and Adolescents: a working group report from the National High Blood Pressure Education Program. National High Blood Pressure Education Program Working Group on Hypertension Control in Children and Adolescents. Pediatrics. 1996;98(4 Pt 1):649-58.

16. Stabelini Neto A, Campos W, Santos GC, Mazzardo Junior O. Metabolic syndrome risk score and time expended in moderate to vigorous physical activity in adolescents. BMC Pediatr. 2014;14(1):1-6.

17. Castro-PineroJ,Laurson KR,Artero EG, Ortega FB, Labayen I, Ruperez AI, et al. Muscle strength field-based tests to identify European adolescents at risk of metabolic syndrome: The HELENA study. J Sci Med Sport. 2019;22(8):929-34.

18. Tanner JM. Growth at Adolescence, 2nd ed. Blackwell, Oxford, 1962.
19. Bouchard C, Tremblay A, Leblanc C, Lortie G, Savard R, Thériault G. A method to assess energy expenditure in children and adults. Am J Clin Nutr. 1983;37(3):461-7.

20. Bratteby LE, Sandhagen B, Fan H, Samuelson G. A 7-day activity diary for assessment of daily energy expenditure validated by the doubly labelled water method in adolescents. Eur J Clin Nutr. 1997;51(9):585-91.

21. Martínez-Gómez D, Wärnberg J, Welk GJ, Sjöström M, Veiga OL, Marcos A. Validity of the Bouchard activity diary in Spanish adolescents. Public Health Nutr. 2010;13(2):261-8.

22. Langlois J, Omorou AY, Vuillemin A, Briancon S, Lecomte E. Association of socioeconomic, school-related and family factors and physical activity and sedentary behaviour among adolescents: multilevel analysis of the PRALIMAP trial inclusion data. BMC Public Health. 2017;17(1):175.

23. Agostinis-Sobrinho C, Gomez-Martinez S, Nova E, Hernandez A, Labayen I, Kafatos A, et al. Lifestyle patterns and endocrine, metabolic, and immunological biomarkers in European adolescents: The HELENA study. Pediatr Diabetes. 2019;20(1):23-31.

24. Canabrava KLR, Amorim PRS, Miranda VPN, Priore SE, Franceschini SCC. Comportamento Sedentário e risco cardiovascular em crianças: Uma revisão sistemática. Rev Bras Med do Esporte. 2019;25(5):433-41.

25. Young DR, Hivert MF, Alhassan S, Camhi SM, Ferguson JF, Katzmarzyk PT, et al. Sedentary Behavior and Cardiovascular Morbidity and Mortality: A Science Advisory From the American Heart Association. Circulation. 2016;134(13):e262-79.

26. Pulsford RM, Blackwell J, Hillsdon M, Kos K. Intermittent walking, but not standing, improves postprandial insulin and glucose relative to sustained sitting: A randomised crossover study in inactive middle-aged men. J Sci Med Sport. 2017;20(3):278-83.

27. Staiano AE, Harrington DM, Broyles ST, Gupta AK, Katzmarzyk PT. Television, adiposity, and cardiometabolic risk in children and adolescents. Am J Prev Med. 2013;44(1):40-47

28. Cho KO, Lee S, Kim YS. Physical Activity and Sedentary Behavior Are Independently Associated with Weight in Korean Adolescents. J Lifestyle Med. 2014;4(1):47-54.

29. Pate RR, Neill JRO, Lobelo F. The Evolving Definition of “'Sedentary ." Exerc Sport Sci Rev. 2008;29208(4):173-8.

30. Chaput JP, Saunders TJ, Mathieu MÈ, Henderson M, Tremblay MS, O'Loughlin J, et al. Combined associations between moderate to vigorous physical activity and sedentary behaviour with cardiometabolic risk factors in children. Appl Physiol Nutr Metab. 2013;38(5):477-83.

31. Fröberg A, Raustorp A. Objectively measured sedentary behaviour and cardio-metabolic risk in youth: A review of evidence. Eur J Pediatr. 2014;173(7):845-60.

32. Kumar B, Robinson R, Till S. Physical activity and health in adolescence. Clin Med (Lond). 2015;15(3):267-72.

33. Physical Activity Guidelines Advisory Committee. 2018 Physical Activity Guidelines Advisory Committee Scientific Report. Washington, DC: United States. 2018.

Recebido: $11 / 11 / 2019$

Aprovado: 27/08/2020

Como citar este artigo:

Santos GC, Campos W, Faria WF, Silva JM, Bozza R, Mascarenhas LPG, Ulbrich AZ, Stabelini Neto A. Rev Bras Ativ Fís Saúde. 2020;25:e0132. DOI: 10.12820/rbafs.25e0132 